\title{
Registration-Based Interpolation Using a High-Resolution Image for Guidance
}

\author{
Graeme P. Penney ${ }^{1}$, Julia A. Schnabel ${ }^{1}$, Daniel Rueckert ${ }^{2}$, David J. Hawkes ${ }^{1}$, and \\ Wiro J. Niessen ${ }^{3}$ \\ ${ }^{1}$ Imaging Sciences Division, Guy's, King's and St. Thomas' Schools of Medicine, King's \\ College London, UK. graeme. penney@kcl . ac.uk \\ ${ }^{2}$ Visual Information Processing Group, Department of Computing, Imperial College London, \\ UK. \\ 3 Image Sciences Institute, University Medical Center Utrecht (UMCU), The Netherlands.
}

\begin{abstract}
A method is presented for interpolation between neighbouring slices in a grey-scale tomographic data set, when a high-resolution image of the same patient is also available. Spatial correspondence between adjacent slices in the high-resolution image are established using a voxel-based non-rigid registration algorithm. These spatial correspondences are used to calculate a set of vectors, which are transferred from the high-resolution image to the lower resolution image by rigidly registering the image volumes together. Linear interpolation is then carried out along these vector directions between neighbouring slices in the low-resolution image. This method has been compared to standard linear interpolation, shape-based interpolation and registration-based interpolation in MR head volumes. Results using a mean square difference error measure show that the proposed method outperforms the other three interpolation techniques.
\end{abstract}

\section{Introduction}

Three-dimensional medical imaging devices typically produce images as a set of slices. The distance between neighbouring pixels in a slice is often smaller than the centre-tocentre slice separation, and therefore the voxel dimensions are generally not isotropic. In a number of image processing, analysis and visualization tasks it is advantageous to have voxel dimensions which are close to isotropic.

This paper describes a method which can use information from a high-resolution image to aid in the interpolation of a low-resolution image. Example clinical scenarios include: functional MR, where a high-resolution anatomical scan is typically acquired before a number of lower resolution functional images; and studies which involve both CT and MR imaging, where a large CT slice thickness has been used to keep the radiation exposure to the patient at an acceptable level. The terms high- and low-resolution used in this paper refer specifically to the resolution in the image ' $Z$ ' direction, i.e. the direction perpendicular to the image slices.

Interpolation techniques can be divided into two groups: scene-based and objectbased methods [4]. In scene-based methods the interpolated intensity is determined only from the image intensities: examples include nearest neighbour, linear and spline-based 
interpolation. These methods can produce large artifacts when the in-plane position of anatomical features shift considerably between slices.

In object-based interpolation some additional information is extracted from the images, and used to help guide the interpolation process. These techniques can be further subdivided into methods which operate on extracted features, and into methods which operate on image intensities. An example of object-based interpolation which uses features is shape-based interpolation [612] which has been extended by allowing registration between slices [7] and using feature guidance [9]. These techniques provide a practical way to interpolate between segmented slices. However, they are unable to interpolate grey-scale images. An example of an object-based method which directly uses the image intensities is the extension to shape-based interpolation proposed by Grevera and Udupa [3]. There are also published methods where non-rigid registration is used to register adjacent slices, and then interpolation is carried out between corresponding positions in each slice [2]. We have recently shown how a registration-based method can outperform both linear and shape-based interpolation [11]. This paper describes an extension to our registration-based method which makes use of information from a high-resolution image to guide the interpolation process.

\section{Description of Algorithm}

For registration-based interpolation two assumptions are made: that adjacent slices contain similar anatomical features, and that the registration algorithm is capable of finding the transformation which maps these similar features together. If the first assumption is violated, and an anatomical feature disappears from one slice to the next, then the advantages of a registration-based approach will be lost. The second assumption is concerned with the types of transformation that the registration algorithm is capable of. If the transformation between features in adjacent slices is outside of the capabilities of the registration algorithm, then the results will be sub-optimum. Examples for this would be: if the underlying model assumes a smooth deformation field, whereas the "true" transformation requires a discontinuity; or if the transformation required by the algorithm is outside of its capture range.

The inputs into the algorithm are shown at the top of Figure 10 a high-resolution (HR) image (a) and a low-resolution (LR) image (b). Figure1(c) shows a schematic of a zoomed region defined as lying inside the white rectangle in (a). Some major image features, such as the brain and skull boundary, are represented by the thick lines and the HR slice planes are represented by the dotted lines. The algorithm proceeds as follows:

1. Each slice in the HR image is registered to the adjacent slice using a 2D voxel-based non-rigid registration algorithm (described in section 2.1). This produces a non-rigid transformation $T_{N R_{i}}$ for each pair of slices $i, i+1$ (where $i=1, \ldots, N_{H R}-1$ and $N_{H R}$ is the number of slices in the HR image). The $T_{N R_{i}}$ are used to calculate a set of interpolation lines $L$. These lines connect the centre of each pixel $\mathbf{u}$ in slice $i$ with its corresponding position, $T_{N R_{i}}(\mathbf{u})$ in slice $i+1$, see Figure 1 (c).

2. The HR and LR images are rigidly registered together to calculate $T$. This transformation is used to transform two adjacent LR slices and an interpolation plane into the HR coordinate system, as shown in Figure1(d). 


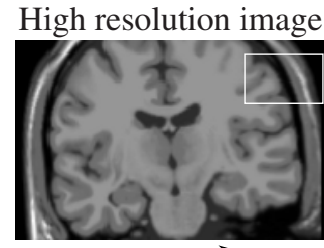

(a)

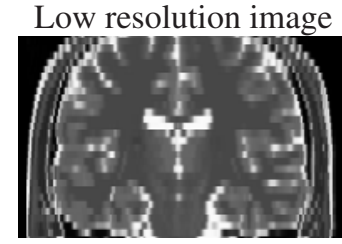

(b)

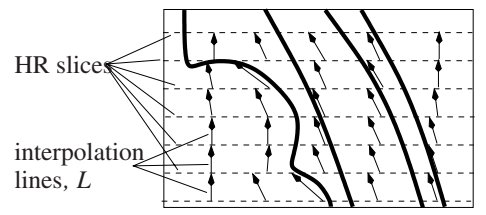

(c)

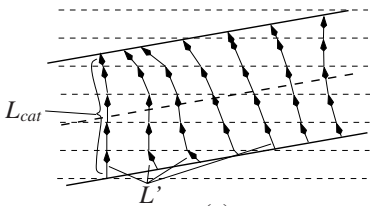

(e)

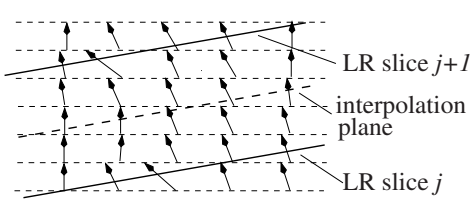

(d)

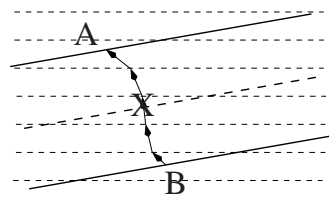

(f)

Fig. 1. Interpolation method: low-resolution (LR) image (b) is interpolated using prior information from high-resolution (HR) image (a). Adjacent HR slices are registered together to give interpolation lines (c). Adjacent LR slices are transformed, using T, into the HR image (d). The interpolation lines are concatenated from LR slice $j$ to LR slice $j+1$ (e). The intensity value at $\mathbf{X}$ is calculated using linear interpolation between the intensity values at $\mathbf{A}$ and $\mathbf{B}$ (f).

3. For each pixel in LR slice $j$ the closest interpolation line is calculated. This line is then translated (parallel to the HR slice planes) so that it intercepts this pixel. These lines form set $L^{\prime}$.

4. The non-rigid transformations $T_{N R_{i}}$ are then successively applied to each interpolation line in $L^{\prime}$ to extend it from LR slice $j$ to LR slice $j+1$. This produces a set of concatenated lines $L_{\text {cat }}$ as shown in Figure 1(e).

5. To interpolate a value at position $\mathbf{X}$ on the interpolation plane, the closest concatenated line in $L_{\text {cat }}$ is found, and translated parallel to the LR slices until it intercepts $\mathbf{X}$. This line is used to calculate positions $\mathbf{A}$ and $\mathbf{B}$ in Figure 1(f).

6. The pixel intensity value at $\mathbf{X}$ is calculated using linear interpolation between the intensity values at $\mathbf{A}$ and $\mathbf{B}$.

This new approach has two main advantages over standard registration-based interpolation: Firstly, the standard approach assumed that features in adjacent slices were connected by straight lines, whereas this method follows the path of connected features in the HR image. Secondly, because the HR slices are closer together there is less chance of the non-rigid registration algorithm finding a local minimum.

Potential disadvantages of the new approach are: Firstly, that errors may be introduced by the rigid registration $T$. Secondly, the interpolation method requires a number 
of non-rigid transformations to be concatenated, and so the overall error will be an aggregate of the individual non-rigid registration errors.

\subsection{Matching Process}

The registration algorithm is a two-dimensional version of the non-rigid registration algorithm of Rueckert et al. [13]. The algorithm models deformations based on a set of B-splines controlled by a regular lattice of control points. The similarity measure was normalized mutual information, and no regularization was used ( $\lambda$ in [13] set to zero).

Three parameters define the optimization process: step size $(s)$ to calculate the finite difference values; grid spacing $(g)$ between the control points; and the maximum number of iterations. The following pairs of $g$ and $s$ values were used for all the experiments described in this paper: $g=20$ and $s=5, s=2.5, s=1.25, s=0.625$; then $g=10$ and $s=5, s=2.5, s=1.25, s=0.625$; then $g=5$ and $s=5, s=2.5, s=1.25$, $s=0.625$ (all values are in $\mathrm{mm}$ ). For each pair of $g$ and $s$ values, when either a minimum of the similarity measure is found, or more than 20 iterations occur, the algorithm then moves along to the next pair of $g$ and $s$ values.

\section{Experimental Evaluation}

The evaluation of our new interpolation method has been carried out in conformity with the paradigm proposed by Grevera and Udupa [4]. We have compared our new approach with three other interpolation methods: linear interpolation, shape-based interpolation (or more specifically, shape-based averaging [3]) and a registration-based method [11]. Linear intensity interpolation was implemented in a standard fashion. The shape-based algorithm was implemented using information from [3], the weighting between pixel dimensions and intensity values (called the intensity scale factor $\mu$ in [3]) was set equal to 0.25 . Euclidean distance transforms were generated by using the method described in [10]. The registration-based algorithm is described in [11]. It uses the registration algorithm and optimization described in Section 2.1 to obtain corresponding positions in each slice, and then carries out linear interpolation between these positions.

\subsection{Data}

Experiments have been carried out using the BrainWeb simulated Brain Database [8] 11. MR T1, T2 and proton density (PD) images were generated with zero noise and image sizes $181 \times 217 \times 181$ with voxel dimensions $1 \times 1 \times 1 \mathrm{~mm}^{3}$. The T1 image was used as the high-resolution image. To simulate the effect of registration errors, this image was translated and rotated by $5 \mathrm{~mm}$ and $5^{\circ}$ in all six degrees of freedom, and then reformatted in this new coordinate system using trilinear interpolation. Each of the slices from this image were then registered to the neighbouring slice. The T2 and PD images were transformed into low-resolution images by removing three from every four slices, resulting in images with a slice thickness of $1 \mathrm{~mm}$ and a centre-to-centre slice separation

\footnotetext{
${ }^{1}$ http://www.bic.mni.mcgill.ca/brainweb/
} 


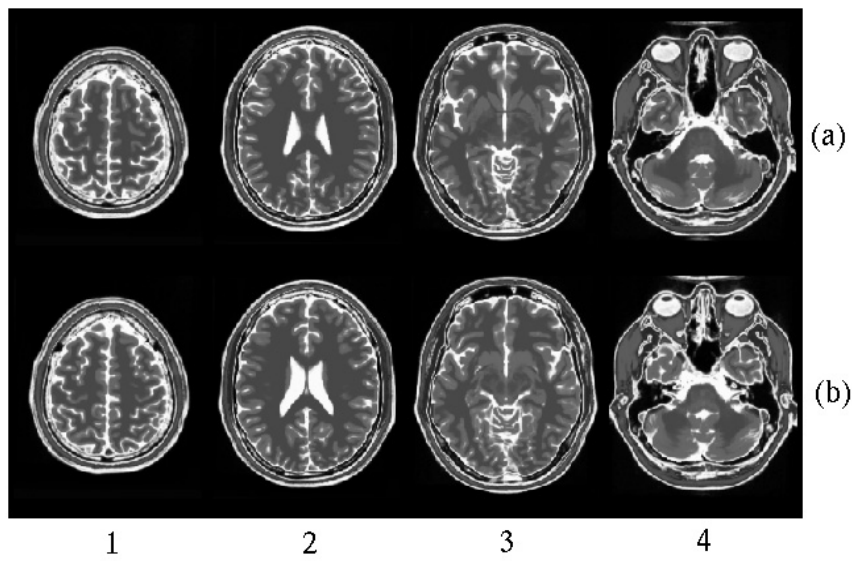

Fig. 2. The four pairs of superior (a) and inferior (b) adjacent slices chosen from the low-resolution $\mathrm{T} 2$ data set.

of $4 \mathrm{~mm}$. Each LR image was then registered to the high-resolution T1 volume, using a rigid registration algorithm based on normalised mutual information [14], to calculate transformation $T$.

\subsection{Method}

Four pairs of adjacent slices were chosen from the low-resolution T2 and PD data sets, as shown in Figure 2. A position was chosen near the top of the head to produce the pair of slices labelled as 1 in Figure 2 Three other pairs of slices were then extracted at $32 \mathrm{~mm}$ intervals below this top pair of slices. Each interpolation algorithm was used to produce a slice midway between the chosen slices. This interpolated slice was then compared to the corresponding slice from the original high-resolution T2 and PD data sets. Initially visual inspection was used to compare the slices, and then the mean square difference (MSD) between the intensity values of the two slices was calculated, as given by Equation (1), where $N_{R}$ is the number of interpolated pixels in the slice, and $I_{i}(\mathbf{u})_{\text {int }}$ and $I_{i}(\mathbf{u})_{\text {orig }}$ are the interpolated and original intensity values at pixel position $\mathbf{u}$.

$$
\operatorname{MSD}=\frac{1}{N_{R}} \sum_{\mathbf{u} \in R}\left(I_{i}(\mathbf{u})_{\text {int }}-I_{i}(\mathbf{u})_{\text {orig }}\right)^{2}
$$

Following Grevera and Udupa [4] we have used a measure called statistical relevance, which essentially shows the percentage difference between two interpolation techniques, e.g. the statistical relevance $r$ between linear $\mathrm{MSD}_{\text {lin }}$ and shape-based averaging $\mathrm{MSD}_{s b a}$ is given by Equation 2 .

$$
r_{s b a / l i n}= \begin{cases}+100 \times\left[1-\mathrm{MSD}_{s b a} / \mathrm{MSD}_{l i n}\right] & , \quad \text { if } \mathrm{MSD}_{l i n}>\mathrm{MSD}_{s b a} \\ -100 \times\left[1-\mathrm{MSD}_{l i n} / \mathrm{MSD}_{s b a}\right] & , \quad \text { otherwise }\end{cases}
$$


Table 1. Statistical relevance values, comparing four interpolation techniques using a mean square difference (MSD) error measure.

\begin{tabular}{|l|c|c|c|c||c|c|c|c||c|}
\hline interpolation & \multicolumn{8}{|c|}{ MSD statistical relevance $r$} \\
methods & \multicolumn{7}{|c|}{ T2 slice number } & \multicolumn{7}{c|}{ PD slice number } & mean \\
& 1 & 2 & 3 & 4 & 1 & 2 & 3 & 4 & value \\
\hline \hline $\mathrm{hrg} / \mathrm{reg}$ & 12.9 & 32.2 & 33.1 & 23.1 & 1.0 & 15.9 & 8.9 & 19.1 & 18.3 \\
\hline $\mathrm{hrg} / \mathrm{sba}$ & 80.4 & 46.1 & 49.8 & 41.9 & 81.9 & 34.4 & 20.9 & 34.5 & 48.7 \\
\hline $\mathrm{hrg} / \mathrm{lin}$ & 85.2 & 53.2 & 49.9 & 46.5 & 88.8 & 53.2 & 32.4 & 48.2 & 57.2 \\
\hline $\mathrm{reg} / \mathrm{sba}$ & 77.5 & 20.5 & 25.0 & 24.4 & 81.7 & 22.0 & 13.2 & 18.9 & 35.4 \\
\hline $\mathrm{reg} / \mathrm{lin}$ & 83.0 & 31.0 & 25.1 & 30.4 & 88.7 & 44.4 & 25.9 & 35.9 & 45.6 \\
\hline sba/lin & 24.7 & 13.2 & 0.1 & 7.9 & 38.1 & 28.7 & 14.6 & 21.0 & 18.5 \\
\hline
\end{tabular}

\section{Results}

Table 1 shows the MSD statistical relevance $(r)$ values which compare the four interpolation algorithms: high-resolution guided (hrg), registration-based (reg), shape-based (sba) and linear (lin). Results are given for both the T2 and PD data sets for each of the four slices individually. All the values in Table 1 are positive, which means that (using MSD as an error measure, and for the data used in this paper) we can rank the interpolation methods from best to worse as: high-resolution guided, registration-based, shape-based and linear.

Figure 3 shows difference or subtraction images for each of the 4 slices in the T2 data set and for each interpolation technique. At the top of the head (slice 1), where the skull diameter changes significantly between slices, large errors were observed when using linear interpolation. Shape-based interpolation accurately interpolated the outer boundary of the skull in this region, but did not perform so well in interpolating structures immediately inside the skull boundary, whereas both the registration-based and highresolution guided methods performed more accurately in this region.

Slice 2 cuts through the ventricles, which can be seen to change greatly in size and shape between the two slices (see Figure 2). Predictably, the linear interpolation algorithm is unable to interpolate these features accurately. The registration-based algorithm also had difficulties, and failed to completely recover the large changes in size and shape of the ventricles. The shape-based algorithm performed well in the region of the ventricles, however, larger differences elsewhere in the images still resulted in a larger MSD value compared to the registration-based method (see Table 11). The best result visually, both near the ventricles and in the surrounding image, was achieved using the high-resolution guided method.

In slices 3 and 4 a general improvement in the interpolation methods can be seen with the images becoming a more uniform grey colour with fewer residual features remaining as you look from the bottom to the top of Figure 3.

\section{Discussion and Future Work}

We have presented a novel interpolation algorithm which can use a high-resolution image to guide the interpolation. Although experiments have only been carried out to interpolate 


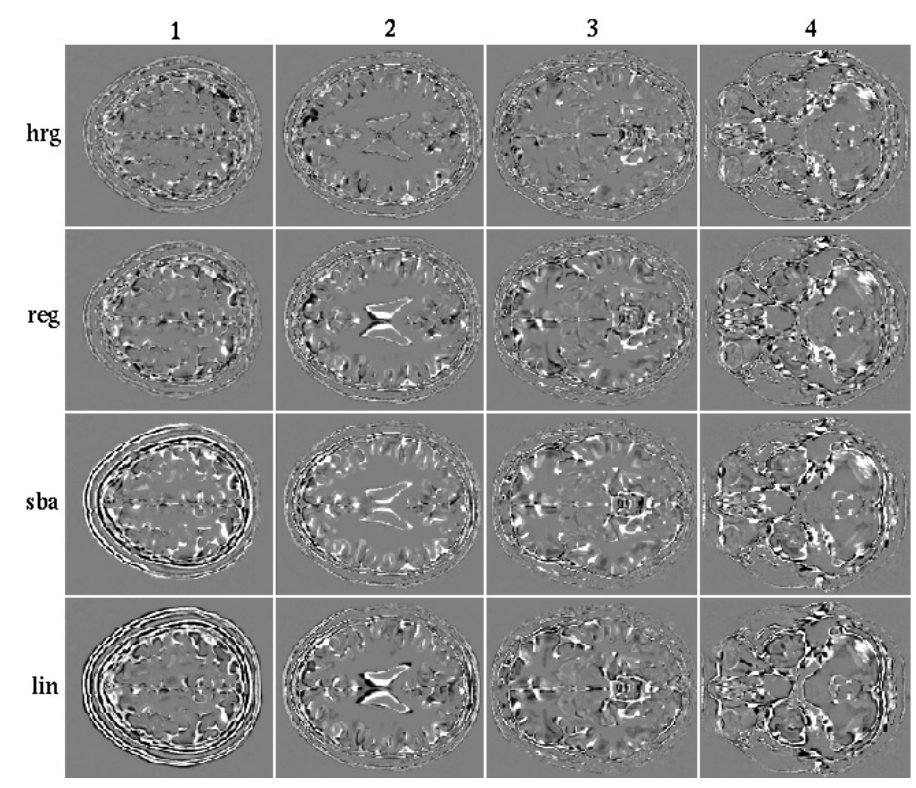

Fig. 3. Difference images $I(u)_{\text {orig }}-I(u)_{\text {int }}$ for each interpolated slice from the T2 image, and for each interpolation technique.

eight slices, these preliminary results are encouraging, showing an average improvement in statistical relevance of 18.3 compared to registration-based interpolation, $48.7 \mathrm{com}$ pared to shape-based averaging and 57.2 compared to linear interpolation.

The algorithm assumes that corresponding structures in adjacent slices from one modality will line up in the same way as corresponding structures from a second modality. This is a reasonable assumption if both of the images are anatomical i.e. MR T1 or T2 weighted, or CT. However, the validity of using a high-resolution T1 image to help interpolate a functional MR image requires further investigation. In addition, problems may arise if the high-resolution image does not depict structures present in the lowresolution image. This could occur if a high-resolution CT image was used to guide the interpolation of soft-tissue structures in a low-resolution MR image.

We have used a very general error measure to compare the interpolation techniques. In future it may be advantageous to tailor the error measure to a particular clinical problem. This type of approach has been proposed by Grevera et al. [5] where, as an example, they examine how interpolation methods influence the accuracy of multiple sclerosis lesion volume measurement.

The algorithm described in this paper requires a rigid-body relationship between the high- and low-resolution images. We are currently working on an algorithm which can allow a non-rigid relationship between the images. This should greatly increase the flexibility of this method. Particularly in areas such as cardiac imaging where it is possible to acquire end-diastolic high-resolution images, where the heart is moving relatively slowly, whereas at other points in the cardiac cycle, as the heart is moving more 
quickly, lower resolution images are usually acquired. We currently believe the greatest benefit of moving to a system which allows a non-rigid relationship between the highand low-resolution images, would be the possibility of replacing the high-resolution image with an atlas image and, therefore, removing the requirement of a high-resolution image altogether.

Acknowledgements. Thanks go to Calvin Maurer Jr. for providing fast distance transfer software; Medical IT - Advanced Development, Philips Medical Systems BV, Best, The Netherlands for funding J.A. Schnabel; and to the Netherlands Organization for Scientific Research (NWO) VENI grant programme for sponsoring W.J.Niessen.

\section{References}

1. D.L. Collins, A.P. Zijdenbos, V. Kollokian, J.G. Sled, N.J. Kabani, C.J. Holmes, and A.C. Evans. Design and construction of a realistic digital brain phantom. IEEE Trans. Med. Imaging, 17(3):463-468, 1998.

2. A. Goshtasby, D.A. Turner, and V. Ackerman. Matching of tomographic slices for interpolation. IEEE Trans. Med. Imaging, 11(4):507-516, 1992.

3. G.J. Grevera and J.K. Udupa. Shape-based interpolation of multidimensional grey-level images. IEEE Trans. Med. Imaging, 15(6):881-892, 1996.

4. G.J. Grevera and J.K. Udupa. An objective comparison of 3-D image interpolation methods. IEEE Trans. Med. Imaging, 17(4):642-652, 1998.

5. G.J. Grevera, J.K. Udupa, and Y. Miki. A task-specific evaluation of three-dimensional image interpolation techinques. IEEE Trans. Med. Imaging, 18(2):137-143, 1999.

6. G.T. Herman, J. Zheng, and C.A. Bucholtz. Shape-based interpolation. IEEE Comput. Graph. Appl., 12(3):69-79, 1992.

7. W.E. Higgins, C. Morice, and E.L. Ritman. Shape-based interpolation of tree-like structures in three-dimensional images. IEEE Trans. Med. Imaging, 12(3):439-450, 1993.

8. R.K.-S. Kwan, A.C. Evans, and Pike G.B. MRI simulation-based evaluation of imageprocessing and classification methods. IEEE Trans. Med. Imaging, 18(11):1085-1097, 1999.

9. T.-Y. Lee and C.-H. Lin. Feature-guided shape-based image interpolation. IEEE Trans. Med. Imaging, 21(12):1479-1489, 2002.

10. C.R. Maurer, Jr., R. Qi, and V. Raghavan. A linear time algorithm for computing exact Euclidean distance transforms of binary images in arbitrary dimensions. IEEE Trans. Pattern Anal. Mach. Intell., 25(2):265-269, 2003.

11. G.P. Penney, J.A. Schnabel, D. Rueckert, M.A. Viergever, and W.J. Niessen. Registrationbased interpolation. IEEE Trans. Med. Imaging. Accepted for publication.

12. S.P. Raya and J.K. Udupa. Shape-based interpolation of multidimensional objects. IEEE Trans. Med. Imaging, 9(1):32-42, 1990.

13. D. Rueckert, L.I. Sonoda, C. Hayes, D.L.G. Hill, M.O. Leach, and D.J. Hawkes. Nonrigid registration using free-form deformations: application to breast MR images. IEEE Trans. Med. Imaging, 18(8):712-721, 1999.

14. C. Studholme, D.L.G. Hill, and D.J. Hawkes. An overlap invariant entropy measure of 3D medical image alignment. Pattern Recognition, 32:71-86, 1999. 